\title{
Cyclical variations in cerebral blood flow velocity
}

\author{
M Y Anthony, D H Evans, M I Levene
}

\begin{abstract}
Because little is known about spontaneous changes in cerebral blood flow in neonates, a newly developed online Doppler technique was used to insonate continuously the middle cerebral arteries of a group of sick $(n=20)$ and full term healthy $(n=16)$ newborn infants for a period of one minute. A total of 290 recordings of epochs each lasting one minute were analysed, and pronounced regular, cyclical variations were seen in the velocity traces of these infants. The cycles occurred 1.5-5 times/minute and were present for at least one epoch in all 20 of the sick infants and in 15 of the 16 healthy mature neonates. Simultaneous recordings of the systemic blood pressure in the sick infants rarely showed the same cyclical variations. The cyclical variation is different from the beat to beat variability seen in the waveforms previously described, and is an additional factor to account for the wide variation in 'normal' velocity recordings obtained when Doppler ultrasound is measured over a short period of time.
\end{abstract}

Measurements of cerebral blood flow velocities by Doppler ultrasound are being increasingly used to study the neonatal cerebral circulation. The information obtained may help us to understand the pathophysiological processes that predispose to neonatal cerebral injury. We have previously reported the development of an online Doppler technique in which the transducer can be applied directly to the skin overlying the middle cerebral artery. The cerebral blood flow velocity is then measured continuously for a period of one minute. ${ }^{12}$ Many previously reported studies have used intermittent, short duration Doppler monitoring; this can analyse only 30 cardiac cycles of data at most. In some of these methods errors have been caused by movement of the Doppler transducer during the studies, and inconsistency in the angle of insonation between the observations. The use of continuous monitoring of velocity for a long period of time seems to provide new information on the variability of the cerebral blood flow velocity signal that has not been previously reported to our knowledge.

\section{Patients and methods} DOPPLER ULTRASOUND

The Doppler ultrasound system used in this study is a modification of the method we have previously described. ${ }^{2}$ A 4 or $6 \mathrm{MHz}$ Doptek pulsed wave button transducer (11 $\mathrm{mm} \times$
$7 \mathrm{~mm} \times 5 \mathrm{~mm}$ ) was fixed to the skin over the baby's temporal bone at the point where the Doppler signal from the ipsilateral middle cerebral artery (MCA) was maximal. A soft Stomahesive wafer (ConvaTec Ltd) was applied to the skin of the temples as this clings without the need for additional adhesive. The transducer was then secured to the wafer with collodion, the Stomahesive protecting the infant's delicate skin from the collodion. The Doppler sample volume, depth, and length was then adjusted electronically to optimise the signal. Once in place the transducer can remain fixed for up to four hours before being removed.

The probe was connected by a fine coaxial cable to a Doptek pulsed wave Doppler unit, which in turn was connected to a digital signal processing unit and a Nimbus microcomputer. The computer automatically switched the system on at predetermined intervals (in this case every 20 minutes) and gathered 60 seconds of both Doppler signals and (in babies with indwelling arterial lines) blood pressure signals. It was possible to override the automatic timing sequence and capture 60 seconds of data at will. The digital signal processing unit carries spectral analysis on the Doppler signal in real time and stores a new range together with the corresponding blood pressure reading every $12.5 \mathrm{~ms}$. The data are then analysed beat by beat to produce a list of values including the systolic, mean, and diastolic values of the maximum frequency envelope and the systolic, mean, and diastolic values of blood pressure for each cardiac cycle. These values were then written to floppy disk and graphed on the Nimbus screen.

The whole unit is transportable and can be wheeled to the cot side. The study was approved by the hospital ethics committee, and informed consent was obtained from at least one parent before each study.

\section{PATIENTS}

Two groups of infants were studied: those who required neonatal intensive care (group 1) and a cohort of normal healthy newborn babies who were nursed on the postnatal ward with their mothers (group 2).

The infants in group 1 all had indwelling arterial catheters or cannulas for clinical monitoring before entry into the study so that continuous blood pressure readings could be obtained. All babies in this group required mechanical ventilation at the time of the first study. A morphine infusion or pancuronium, or both, were used when clinically indicated. All the infants were continuously monitored with either an indwell- 
Table 1 Details of sick infants

\begin{tabular}{lcc}
\hline $\begin{array}{l}\text { Gestational age } \\
\text { (weeks) }\end{array}$ & No of infants & $\begin{array}{l}\text { Weight range } \\
(\mathrm{g})\end{array}$ \\
\hline$\leqslant 30$ & 12 & $780-1220$ \\
$31-34$ & 7 & $1040-2170$ \\
$>34$ & 1 & 2820 \\
\hline
\end{tabular}

Table 2 Details of studies of cerebral blood flow velocity

\begin{tabular}{lcl}
\hline & Group 1 & Group 2 \\
\hline No of babies studied & 20 & 16 \\
No of studies carried out & 32 & 16 \\
No of 1 minute epochs & 241 & 49 \\
No of babies with cyclical variations & 20 & 15 \\
No (\%) of epochs containing cyclical & $149(62)$ & $30(61)$ \\
variations & 64 & 52 \\
Maximum percentage variability & $10-64$ & $11-52$ \\
Range of percentage systolic change & 23 & 24 \\
\hline Median percentage systolic change & & \\
\hline
\end{tabular}

ing arterial oxygen electrode or an oxyhaemoglobin saturation monitor that was applied across the infant's hand. Arterial blood gas tensions were measured before and after each study.

\section{Results}

A total of 36 infants were studied, 20 in group 1 and 16 in group 2 . The median gestational age of the sick infants in group 1 was 29 weeks (range 24-37), and their median birth weight was $1150 \mathrm{~g}$ (range 780-2820). Twelve of the infants in group 1 were 30 weeks' gestational age or less (see table 1). Eleven babies were studied on one day only, six on two separate days, and three on three separate days, making a total of 32 studies. The postnatal ages at the start of each study ranged from 2 hours to 8 days. The length of any one study on a baby ranged from one and a half hours to four hours depending on the quality of the Doppler signal and the baby's clinical condition.

All the infants in group 2 were healthy. Their gestational ages ranged from 37-42 weeks (median 39) and they were all studied at postnatal ages ranging from 1-7 days. All the infants in group 2 were appropriate weights for their gestational ages. No healthy infant was studied on more than one occasion and no study lasted more than two hours. The median number of epochs recorded on each infant in group 2 was three (range 1-6). The infants had been fed 1-2 hours before the study, and most were either asleep or in a quiet awake state.

A total of 290 epochs each lasting 60 seconds were analysed. Visual examination of the one minute tracing showed pronounced variability over the course of the recording. There were two types of variability: the first was the beat to beat variability that was described by Perlman $e t$ $a l$ and is illustrated in fig $1,{ }^{3}$ and the second was a pattern of regular cyclical variation in the signal with a frequency of between 1.5 and $5 /$ minute (fig 2). We referred to this pattern as 'cycling'. Most babies had both patterns within the same recording.

The frequency of the cycling was counted as the number of phases present within each epoch of one minute. The degree of variability was cal-
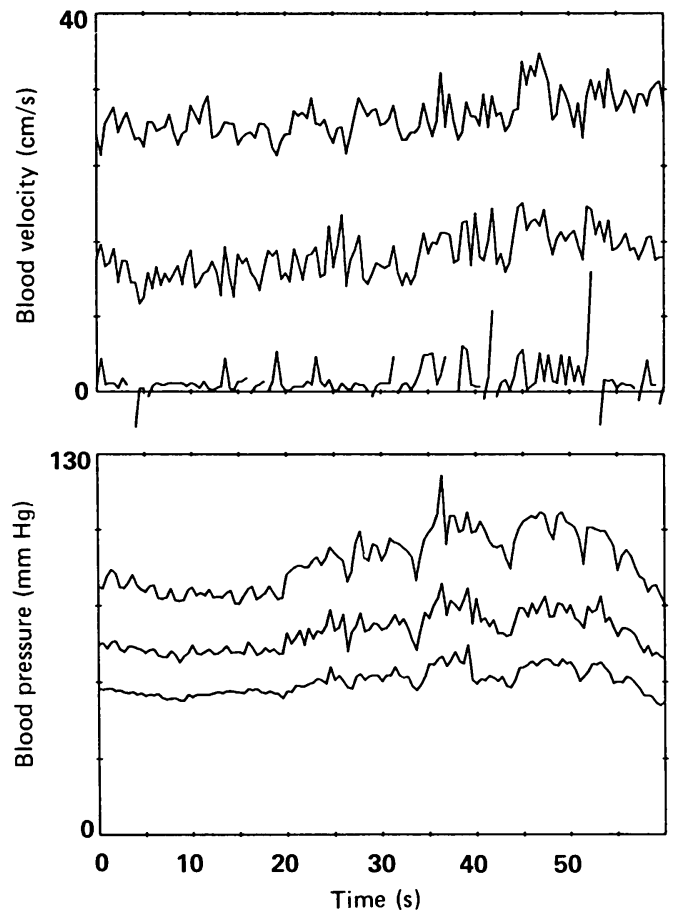

Figure 1 Marked beat to beat variability in blood pressure and cerebral blood flow velocity traces.
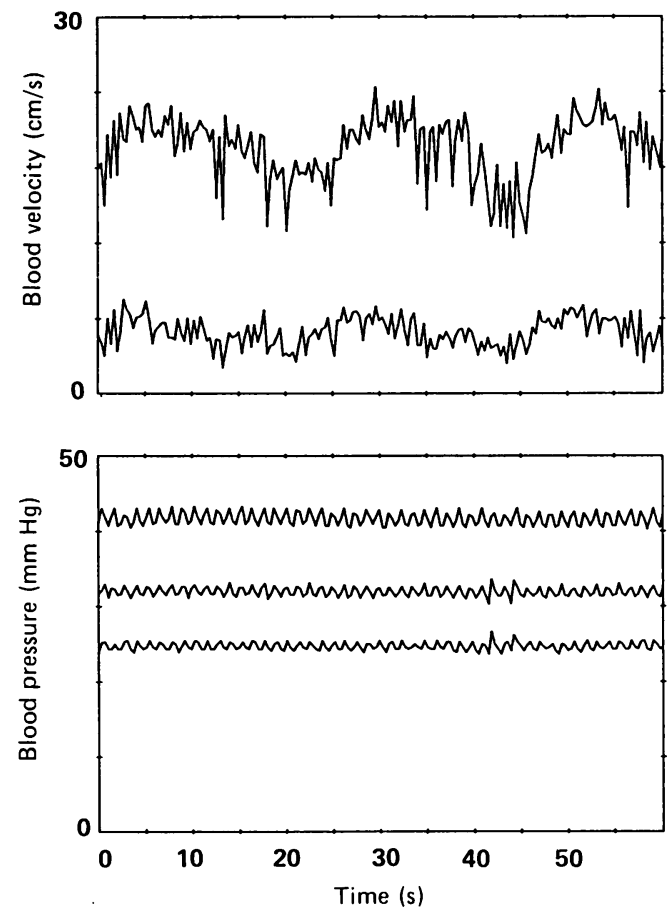

Figure 2 Cyclical variability in cerebral blood flow velocity at 3 cycles/minute with superimposed beat to beat variability. The blood pressure trace shows spikes coinciding with the ventilator inflations at 50 breaths/minute. Diastolic cerebral blood flow is zero.

culated by taking the maximum systolic velocity of a cycle (a) and subtracting the minimum systolic velocity of the same phase (b) and dividing by the maximum systolic velocity $((a-b) / a)$. This was expressed as a percentage. Where the beat to beat variation made the value of (a) or (b) obscure, the cycling variability was not measured although the underlying frequency was obvious on inspection (see table 2). 
GROUP 1

All 20 babies in group 1 showed cyclical variation at some time, although the variability and frequency of the cycling varied among babies and even within the same baby during different epochs. Only one baby did not show any cyclical variation during one complete study of one and a half hours (six epochs), but did show cycling changes during a study the following day. Only two of the 20 babies had cyclical variation during every 60 second epoch, although these were in the shorter studies.

Of the 241 epochs analysed, cycling episodes occurred in $149(62 \%)$. The frequency varied from 1.5 to 5 cycles a minute; this refers both to individual babies at different epochs and among the different babies. In some babies cyclical variation was not present during one epoch but was seen during the next epoch 20 minutes later.

The cycling variability of the systolic velocity could be calculated in 103 epochs and ranged between $10 \%$ and $64 \%$ (median $23 \%$ ). The cycling variability altered from baby to baby and from epoch to epoch within the same baby. Among the infants in group 1 there seemed to be no correlation between the presence or frequency of cycling or the gestational age or the severity of the infant's illness. Out of the total of 32 studies, infants received pancuronium alone $(n=8)$, morphine alone $(n=11)$, both $(n=2)$, or neither $(n=11)$. There were no differences in the number or variability of cycles when the use of these two drugs was compared.

In many cases the blood pressure recordings made at the same time as the cerebral blood flow velocity recordings did not show any variability (fig 2). In eight studies there was pronounced beat to beat variability (fig 1 ) and all the ventilated babies showed increases in blood pressure
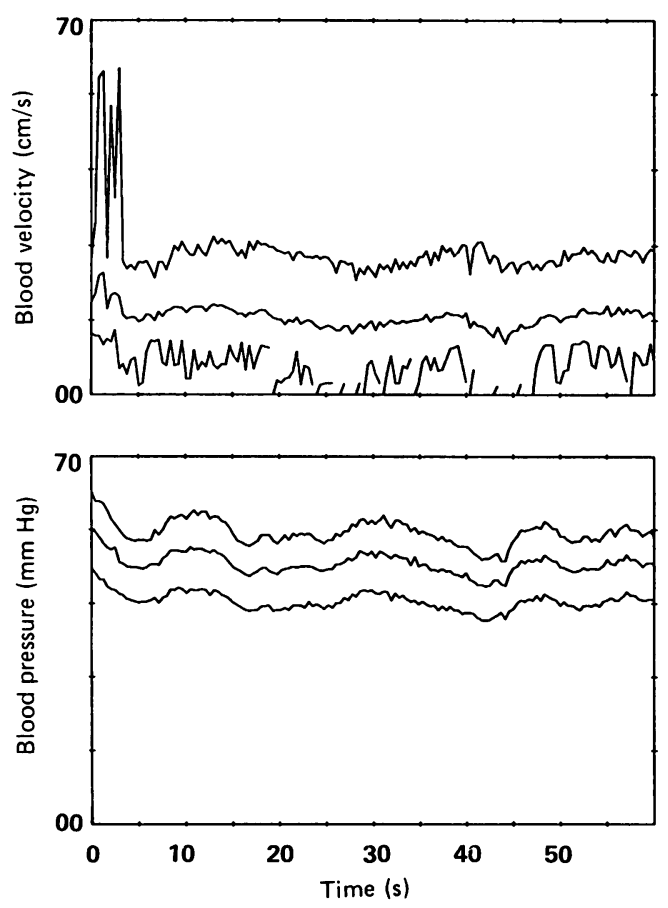

Figure 3 Cyclical variability of both cerebral blood flow velocity and blood pressure at different frequencies. that coincided with the ventilator inflations (fig 2). Cycling was seen on the blood pressure traces of seven babies (fig 3). This usually occurred during more than one epoch in each of the babies who showed this cyclical blood pressure change, but none showed it continuously. Of the seven babies, two were well breathing air on low rate ventilation without sedation, and five were sedated with morphine or pancuronium and had high ventilation requirements; one of the five was also receiving a dopamine infusion. When cycling was seen in both blood pressure and cerebral blood flow velocity recordings the cycles were never in exact phase nor of the same frequency (fig 3).

We could find no relationship between the cycling seen in the Doppler traces and any of the other variables measured. In particular, there were no significant differences in the infant's heart rate measured at the highest and lowest systolic phases of the cycle. There were no consistent changes in arterial oxygen concentration or haemoglobin saturation recordings during the course of the cycles, which might have accounted for the variations seen. The cycling was not abolished by the infusion of plasma.

\section{GROUP 2}

Of the 16 babies in this group, 15 showed cycling at some time, and the frequency varied from 1.5 to $5 /$ minute. A total of 49 epochs were studied of which 30 showed cycling (61\%). These healthy term infants showed the same degree of beat to beat variability in their velocity traces as the sick and preterm infants.

The cycling variability could be calculated in 20 cycles. The median percentage systolic change during cycling was $24 \cdot 2 \%$ (range $10 \cdot 7$ $51.6 \%$ ). The heart rate did not alter during the phase of the cycles. Cycling occurred in infants who were either awake or asleep. It was not possible to measure blood pressure directly in this group and indirect oscillometric methods for recording blood pressure were not possible because they are intermittent, and when the cuff was inflated the infant was disturbed.

There was no difference in the variability between the sick and the full term healthy infants $(p=0 \cdot 11)$.

\section{Discussion}

This study shows that there is a common pattern of regular cyclical variation most clearly seen in systolic cerebral blood flow velocity and occurring at a frequency of 1.5 to 5 cycles/ minute. The cycles are present in both sick extremely preterm and healthy full term neonates during the first eight days of life. The babies that we studied cover a wide range of gestational ages and were studied up to eight days of postnatal life. We do not have data beyond eight days, and are unaware of any studies containing such data.

Cowan has described this cyclical variability in 18 healthy, 5 day old, full term neonates undergoing sleep studies, ${ }^{4}$ but she could not relate her findings to rapid eye movement sleep 
nor to abnormality on the electroencephalogram. Not all the babies in our study were sleeping, and cycling was also present in those who were awake. Two studies of the adult cerebral circulation using periods of continuous Doppler recording lasting up to 10 minutes did not report similar cycling variability. ${ }^{56}$

Two infants in group 1 had haemodynamically important patent ductus arteriosus and required treatment with indomethacin. In neither case was a diastolic component of the sonogram recorded. Five other infants in group 1 also had heart murmurs consistent with patent ductus arteriosus but were not thought to have a haemodynamically important lesions; no infant in group 2 had a cardiac murmur. The cycling pattern was seen at some time in all but one of the 36 infants studied and there were no differences in either the frequency or the amplitude of the cycles between babies with and without murmurs or the two with symptomatic patent ductus arteriosus. It is most unlikely that a patent ductus has any influence on the cycling phenomenon.

There are two possible causes of these cycles: local factors associated with vessels and cerebral events. The diameter of the vessels, and therefore blood velocity, is influenced by various local factors including the arteriolar concentration of carbon dioxide, catecholamine concentrations, neural factors, and myogenic reflexes within the vessel wall. ${ }^{7}$ Among the sick infants studied here, many had been given a nondepolarising muscle blocker (pancuronium) to inhibit spontaneous respiration. There were no changes in ventilator rate during epochs, and it is therefore unlikely that there was a cyclical change in the carbon dioxide tension that caused the regular changes in cerebral blood flow velocity that we observed.

Little is known about the fine control mechanisms of the cerebral vasculature. For autoregulation to occur there must be a feedback mechanism that causes a change in arteriolar size in response to changes in the cerebral blood flow. In most of the infants studied there was no change in blood pressure at the time of the regular cycling cerebral blood flow velocity pattern. It seems unlikely that the effect we saw was the result of a passive myogenic response secondary to systemic changes in either cardiac output or peripheral resistance.

It is possible that this is a fairly primitive phenomenon and we suggest that the cycling in velocity may be an immature response that represents an underdamped autoregulatory mechanism. The cycles may represent rhythmical changes in cerebral vascular tone with a 'hunting' effect on the underlying cerebral blood flow velocity and presumably on cerebral blood flow. This may disappear with cerebral maturation, but it will be of interest to study older infants with this technique to discover at what age the cycling disappears. Although it is most likely that the small cerebral arterioles are responsible for the cycling, it is also conceivable that there may be rhythmic changes in tone of the major cerebral arteries. There is some evidence that in adults the major cerebral arteries play a part in cerebral autoregulation. ${ }^{8}$ The
Doppler technique will not be able to distinguish changes in velocity arising as a result of either large or small arteriolar changes.

One must also consider that the cyclic activity may also be the result of more widespread cerebral events such as seizures or cerebral oedema. Seizures are associated with pronounced changes in cerebral blood flow and intracranial pressure. It is possible that cyclical changes in cerebral blood flow velocity may be the result of seizure activity, but were not found by Cowan who made continuous electroencephalographic recordings during her Doppler studies. ${ }^{4}$ As these changes were also seen in 15 out of 16 healthy full term infants, seizures are most unlikely to have been the cause. Lundberg described cyclical changes in intracranial pressure at $0.5-2$ cycles/minute in adults and referred to them as ' $B$ ' waves. ${ }^{9}$ These changes were of irregular amplitude and interval and were associated with stupor or coma. If the patients were aroused the B waves became less regular or disappeared. They were also associated with Cheyne-Stokes breathing and would disappear once the patient was ventilated. We have also seen regular fluctuations in intracranial pressure in critically ill full term asphyxiated infants. ${ }^{10}$ The frequency of these fluctuations was about 0.5 cycles/minute, but all the infants were extremely ill and all died. Cerebral oedema is also most unlikely to have caused the cyclical variation in our babies, none of whom had severe birth asphyxia.

The beat to beat variability described by Perlman et al was measured in the anterior cerebral arteries of premature infants being ventilated for respiratory distress. ${ }^{3}$ This variability was reflected in the simultaneously recorded blood pressure and was associated with an increased risk of intraventricular haemorrhage. The same workers later showed that this variability was abolished by muscle relaxing drugs. ${ }^{11}$ They concluded that lack of synchrony between the infants' respiratory efforts and the ventilator was responsible for the beat to beat fluctuations in both blood pressure and cerebral blood flow velocity. Rennie et al have shown that there is a reduction in the beat to beat variability in cerebral blood flow velocity in ventilated preterm infants once synchronous breathing is established, ${ }^{12}$ and Rennie has shown that the variability can be reduced by cardiovascular support with either dopamine or plasma infusions. ${ }^{13}$ The variability was greatest within the first 12 hours of life and then gradually reduced over the next 36 hours.

The cycling variability that we have described in this report is quite different from the beat to beat variability described by Perlman et $a l$ and Rennie $e t a l$, but the illustrations of the velocity and blood pressure recordings clearly show that this form of beat to beat variability is superimposed on the rhythmical cycling variability at 1.5 to 5 cycles/minute.

The most immediate clinical relevance of this observation with respect to further Doppler studies is in the error that may occur if velocity data are recorded over short periods of time. Almost all published studies that have used Doppler techniques during the neonatal period 
have collected data for 30 consecutive cardiac impulses at most (about 15 seconds). This is one factor in the high intraobserver variability. As both systolic and diastolic frequencies seem to vary to the same degree, cycling variability will be reflected to a much lesser extent by measurements of the resistance or pulsatility indices. These indices correlate less well with changes in cerebral blood flow than measurements of the peak systolic velocity, end diastolic velocity, or area under the velocity curve. ${ }^{14} 15$

MYA was supported by the Spastics Society. We thank Doptek for providing us with the Doppler unit.

1 Evans DH, Schlindwein FS, Levene MI. An automatic system for capturing and processing ultrasonic Doppler signals and blood pressure signals. Clin Phys Physiol Meas 1989;10:241-51.

2 Fenton A, Evans DH, Levene MI. On line cerebral blood flow velocity and blood pressure measurement in neonates: flow velocity and blood pressure measurement in

3 Perlman JH, McMenamin JB, Volpe JJ. Fluctuating cerebral blood flow velocity in respiratory distress syndrome. N Engl F Med 1983;309:204-9.

4 Cowan $F$. Cerebral blood velocity in the sleeping normal newborn infant. Studies on the cerebral circulation of the newborm infant. Oslo: A/S Holstad-Trykk, 1987:107-31. (Thesis.)

5 Hauge A, Thoresen M, Walloe L. Changes in cerebral blood flow during hyperventilation and $\mathrm{CO} 2$-breathing measured transcutaneously in humans by a bidirectional, pulsed, ultrasound Doppler blood velocity meter. Acta Physiol Scand 1980;110:167-73.

6 Ellingsen I, Hauge A, Nicolaysen M, Thoresen M, Walloe L. Changes in human cerebral blood flow due to step changes in $\mathrm{PaO} 2$ and $\mathrm{PaCO} 2$. Acta Physiol Scand 1987;129:157-63.

7 Simpson JA, Fitch W. The cerebral circulation. In: Simpson JA, Fitch W, eds. Applied neurophysiology: with particular JA, Fitch W, eds. Applied neurophysiology: with particular

reference to anaesthesia. London: Wright, 1988:298-315.
$8 \mathrm{McHedlish}$ ili G. Principles of cerebral blood flow control a deductive approach. In: Bevan JA, ed. Arterial behavior and blood circulation in the brain. New York: Consultants Bureau, 1985:17-41

9 Lundberg $\mathrm{N}$. Continuous recording and control of ventricular fluid pressure in neurosurgical practice. Acta Psychiatrica et Neurologica Scandinavica 1960;36 (supp 149):1-193.

10 Levene MI. Neonatal neurology. Edinburgh: Churchill Livingstone, 1987.

11 Perlman JH, Goodman S, Kreusser KL, Volpe JJ. Reduction in intraventricular hemorrage by elimination of fluctuating cerebral blood flow velocity in preterm infants with respiratory distress syndrome. $N$ Engl f Med 1985;312:1353-7.

12 Rennie JM, South M, Morley CJ. Cerebral blood flow velocity variability in infants receiving assisted ventilation. Arch ity variability in infants recei

13 Rennie JM. Cerebral blood flow velocity variability after cardiovascular support in premature babies. Arch Dis Child 1989;64:897-901.

14 Hansen NB, Stonestreet BS, Rosenkrantz TS, Oh W. Validity of Doppler measurements of anterior cerebral artery blood flow velocity: correlation with brain blood flow in piglets. Pediatrics 1983;72:526-31.

15 Greisen G, Johansen K, Ellison PH, Fredriksen PS, Mali J, Friis-Hansen B. Cerebral blood flow in the newborn infant: comparison of Doppler ultrasound and ${ }^{133}$ xenon clearance. 